\title{
Compressing Electron Pulse with Alternating Electric Field
}

\author{
Chao Wang1, Yifan Kang² \\ ${ }^{1}$ Key Laboratory of Ultra-Fast Photoelectric Diagnostics Technology, Xi'an Institute of Optics and Precision Mechanics, Chinese \\ Academy of Sciences, Xi'an, China \\ ${ }^{2}$ School of Science, Air Force Engineering University, Xi'an, China \\ Email: gooodwang@foxmail.com
}

How to cite this paper: Wang, C. and Kang, Y.F. (2018) Compressing Electron Pulse with Alternating Electric Field. Journal of Applied Mathematics and Physics, 6, 2313-2321.

https://doi.org/10.4236/jamp.2018.611192

Received: June 1, 2018

Accepted: November 19, 2018

Published: November 22, 2018

\begin{abstract}
A novel technique of symmetric type quasi-linear electron pulse duration modulation is proposed. The salient feature from the conventional photoelectron gun is the introduction of the alternating electric field resonator. The electric field that results is synchronously controlled to generate the desired quasi-linear differential energy modulation on the electron pulse passing through. The effect resulted directly is that the leading electrons undergo negative energy modulation and decelerate, while the rear ones positive energy modulation and accelerate, which eventually leads to electron-pulse-duration modulation. The technical details are demonstrated.
\end{abstract}

\section{Keywords}

Electron Pulse, Temporal Broadening, Differential Energy Modulation

\section{Introduction}

Electron-optical methods based on ultrafast manipulation of ultra-short electron pulse have become firmly established as the most advanced in the area of time-resolved events recording and investigation [1]-[12]. Illuminating a photocathode by an optical signal, one can gain an exact transformation of temporal modulation of light power density within the incident optical signal into spatial modulation of charge density within the electron packet. With the criteria of how the photoelectron bunch that generated can be further used, the following two types of techniques can be distinguished. One is related with the problem of measuring the intensity-time profile of incident optical radiation, in which the photoelectron pulse produced is considered as a carrier of information on the incident light pulse structure, and all subsequent transformations of the bunch 
within the system are aimed at preserving and then revealing that information upon the screen. Streak tube is typical of this kind of applications [5] [11]. In the other, however, the photoelectron packet from photoemission is not considered as the information carrier of incident optical radiation but as a probing tool for subsequent applications. Thus the main purpose is to gain an electron pulse with controllable duration. Ultrafast electron diffraction (UED) is the application representative [8]. Whether for streak camera or for UED, the time-resolved performance is characteristic of its applications and attracting more attention almost ever since their discovery. Currently there still remains a big technological challenge in improving time-resolved performance of both UED and streak camera. For the former, temporal broadening of electron pulse induced by both space charge effect and its initial energy spread prevents obtaining high-brightness electron pulses with sub-100 fs durations, limiting the range of phenomena that can be studied with this technique.

In this contribution, we propose the method of symmetric type quasi-linear electron pulse duration modulation. The salient feature is the introduction of an alternating electric field resonator into the conventional photoelectron gun. The electric field that results is synchronously controlled to provide the desired quasi-linear differential energy modulation on the electron pulse. With the result that the leading electrons undergo negative energy modulation and decelerate, while the rear ones positive energy modulation and accelerate, the initial energy modulation eventually leads to electron-pulse-duration modulation. The technical details are demonstrated.

\section{Operational Principle of Compression Technique}

\subsection{Differential Energy Modulation with Alternating Electric Field}

The proposed electron-pulse-duration modulation technique is illustrated in Figure 1. The salient feature is the introduction of the "alternating electric field resonator", which provides the appropriate alternating electric field to impose differential energy modulation on electrons among the pulse. The front and the end mesh wall act as the anode and the energy modulation electrode, respectively. The system has cylindrical axially symmetric structure. The transition-type photocathode and the anode mesh form electron pulse accelerating region of axial length $d_{1}$, upon which the voltage $U$ is applied. The differential energy modulation on the electron pulse is achieved in energy modulation region of axial length $d_{2}$ between the two mesh electrodes, while the electron-pulse-duration modulation is finished in the field-free drifting region of axial length L between energy modulation electrode and target plane. The energy modulation region and field-free drifting region altogether constitute the electron pulse modulation region. An axial uniform magnetic field $\mathrm{B}$ is incorporated to confine electrons pulse radically throughout the whole system.

During the transition process of electron pulse in the electron-optical system, the two factors that contribute much to its pulse broadening are well known as 
the initial energy spread of photoelectron from photocathode and the inherent space charge effect. As for the latter, the electrons in leading part are subject to an overall accelerating force, while the rear ones decelerating force. The joint influence from both is the transition time dispersion between electrons of different part in the electron pulse, which is the essence of electron pulse broadening. In regards of the electron pulse broadening from space charge effect, the analytic or relatively simple calculating program is not yet available. But it has been proved, both theoretical and experimentally, that this kind of broadening possesses the characteristics of pulse center symmetry in comparison with the case without space charge effect, i.e., the leading part and the rear almost have equal broadening magnitude [5]. It is just based on the mentioned feature that, mainly for concise technical descriptions of the proposed method, the temporal broadening from space charge effect is neglected in the following discussion on condition of introducing no any ambiguity on details understanding.

In the electron-optical system of Figure 1, paraxial approximation could be applied for electron motion equation and the axial motion can be described as

$$
\frac{d^{2} z}{d t^{2}}=\eta \phi^{\prime}(z)
$$

in which, origin of coordinate axis $Z$, also the system symmetric axis, is fixed at center of photocathode and points along the motion direction of electron pulse, $z$ indicates the electron position at $t, \phi(z)$ the potential at position of $z$, and $\eta=e / m_{e}$ the ratio of electron charge to mass. Expression (1) steps further as following

$$
\left(\frac{d z}{d t}\right)^{2}=2 \eta[\phi(z)-\phi(0)]+\left.\left(\frac{d z}{d t}\right)^{2}\right|_{t=0} .
$$

Considering the square electron pulse just emitted from photocathode, with initial energy spread of $\Delta \varepsilon_{0}$ and initial pulse duration of $\tau_{0}$, the initial axial

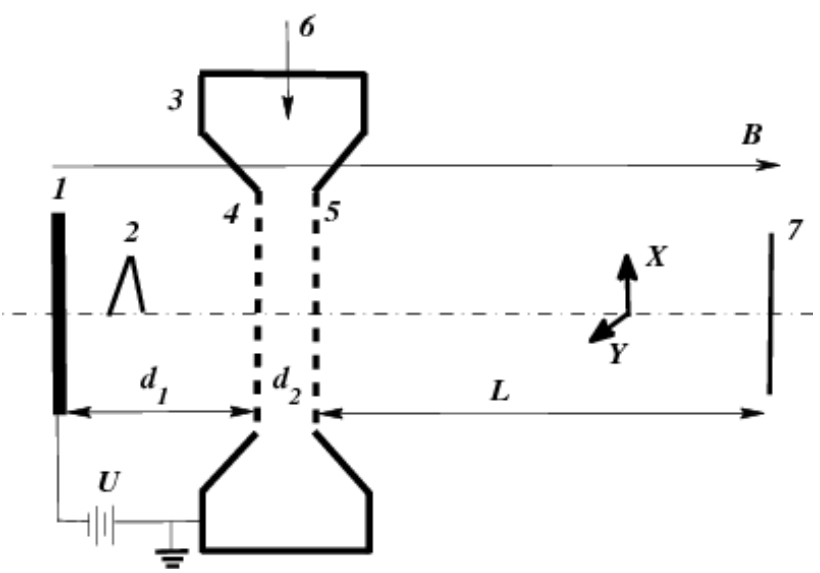

Figure 1. Schematic diagram of the electron-pulse-duration modulation technique using alternating electric field. 1-transition-type photocathode; 2-electron pulse; 3-resonator for alternating electric field; 4-anode mesh (front wall of the resonator); 5-voltage modulation mesh (end wall of the resonator); 6-trigger signal generator, 7-target plane. 
length $I_{0}$ and the axial velocity difference $\Delta v_{0}$ between the electrons in the most leading and the most rear can be given respectively as

$$
\begin{aligned}
& l_{0}=\tau_{0} \sqrt{\frac{2 \Delta \varepsilon_{0}}{m_{e}}}+\frac{\eta U \tau_{0}^{2}}{2 d_{1}}, \\
& \Delta v_{0}=\sqrt{\frac{2 \Delta \varepsilon_{0}}{m_{e}}}+\frac{\eta U \tau_{0}}{d_{1}} .
\end{aligned}
$$

So the electron initial axial energy of the two portions mentioned is approximated as $\varepsilon_{\text {last }}=0$ and $\varepsilon_{\text {first }}=0.5 m_{e} \Delta v_{0}^{2}$. Using the same settings as that in practical engineering, $l_{0}$ is of micrometer scale and can be neglected for electron transition time in photocathode-anode region since it is much smaller than the distance of $d_{1}$ in millimeter scale. Using expression (2), the time for the electron with initial axial energy to fly through the photocathode-to-anode acceleration region turns out to be

$$
t_{1}\left(\varepsilon_{i}\right)=\frac{m_{e} d_{1}}{e U}\left[\sqrt{\frac{2\left(\varepsilon_{i}+e U\right)}{m_{e}}}-\sqrt{\frac{2 \varepsilon_{i}}{m_{e}}}\right],
$$

so the electron pulse duration at the anode mesh is

$$
\tau=t_{1}\left(\varepsilon_{\text {last }}\right)-t_{1}\left(\varepsilon_{\text {first }}\right) .
$$

Apparently, the following relation is established

$$
\tau>\tau_{0},
$$

which indicates the inevitable temporal broadening of electron pulse during the photocathode-to-anode acceleration region, just like that in streak camera or UED.

The alternating voltage of energy modulation electrode relative to anode in the resonator can be assumed to take the general form of $U_{m}(t)=-U_{\max } \sin \left(2 \pi t / T_{m}+\varphi_{0}\right)$. For simplicity the initial phase $\varphi_{0}$ is chose to be 0 , without any hurt on the method detail understanding. The overall transition time for electron with initial axial energy $\varepsilon_{i}$ to fly through the system in Figure 1 can be obtained as

$$
\begin{gathered}
t\left(\varepsilon_{i}\right)=t_{1}\left(\varepsilon_{i}\right)+t_{m}\left(\varepsilon_{i}\right), \\
t_{m}\left(\varepsilon_{i}\right)=t_{2}\left(\varepsilon_{i}\right)+t_{3}\left(\varepsilon_{i}\right), \\
t_{2}\left(\varepsilon_{i}\right)=\frac{m_{e} d_{2}}{e U_{m}\left(t_{1}\left(\varepsilon_{i}\right)\right)}\left[\sqrt{\frac{2\left\{\varepsilon_{i}+e U+e U_{m}\left[t_{1}\left(\varepsilon_{i}\right)\right]\right\}}{m_{e}}}-\sqrt{\frac{2\left(\varepsilon_{i}+e U\right)}{m_{e}}}\right], \\
t_{3}\left(\varepsilon_{i}\right)=\sqrt{\frac{m_{e} L^{2}}{2\left\{\varepsilon_{i}+e U+e U_{m}\left[t_{1}\left(\varepsilon_{i}\right)\right]\right\}}}
\end{gathered}
$$

in which $t_{1}\left(\varepsilon_{i}\right), t_{2}\left(\varepsilon_{i}\right)$ and $t_{3}\left(\varepsilon_{i}\right)$ are the transition time of electrons respectively in acceleration region, energy modulation region and field-free drifting region. Obviously $t_{m}\left(\varepsilon_{i}\right)$ is the transition time in electron pulse modulation region. Considering two electrons with initial axial energy relation of $\varepsilon_{1}<\varepsilon_{2}$, with the following conditions on energy-modulation voltage met, 


$$
\begin{gathered}
\left|U\left(\varepsilon_{1}\right)\right|,\left|U\left(\varepsilon_{2}\right)\right|>\varepsilon_{1}, \varepsilon_{2}, \\
U\left(\varepsilon_{1}\right)>0, \\
U\left(\varepsilon_{2}\right)<0,
\end{gathered}
$$

the following relation is always established

$$
t_{m}\left(\varepsilon_{\text {last }}\right)<t_{m}\left(\varepsilon_{\text {first }}\right),
$$

which means that the compensation for electron pulse broadening emerges.

Figure 2 also shows that, besides the confinement condition mentioned above, the modulation voltage should be limited into the range between the negative and the positive half alternating voltage maximum to achieve the quasi-linear modulation (for $\sin (x) \approx x$ within this interval). Another indispensable condition for symmetric modulation is that transition time of the central electrons through the acceleration region should coincide with the zero point of the alternating voltage used. So the following two relations should be met.

$$
\begin{gathered}
t_{\text {mid }}=\left(k+\frac{1}{2}\right) T_{m}, \\
t_{1}\left(\varepsilon_{\text {first }}\right) \geq\left(k+\frac{5}{12}\right) T_{m} .
\end{gathered}
$$

Here $k=0,1,2, \cdots$. The parameter $k$ is called "alternating voltage nodes", which defines the corresponding time interval for differential energy modulation. Combining (16) with (17), one can obtain the restriction on $k$.

$$
k \leq \frac{6 t_{1}\left(\varepsilon_{\text {first }}\right)-5 t_{\text {mid }}}{12\left[t_{\text {mid }}-t_{1}\left(\varepsilon_{\text {first }}\right)\right]} .
$$
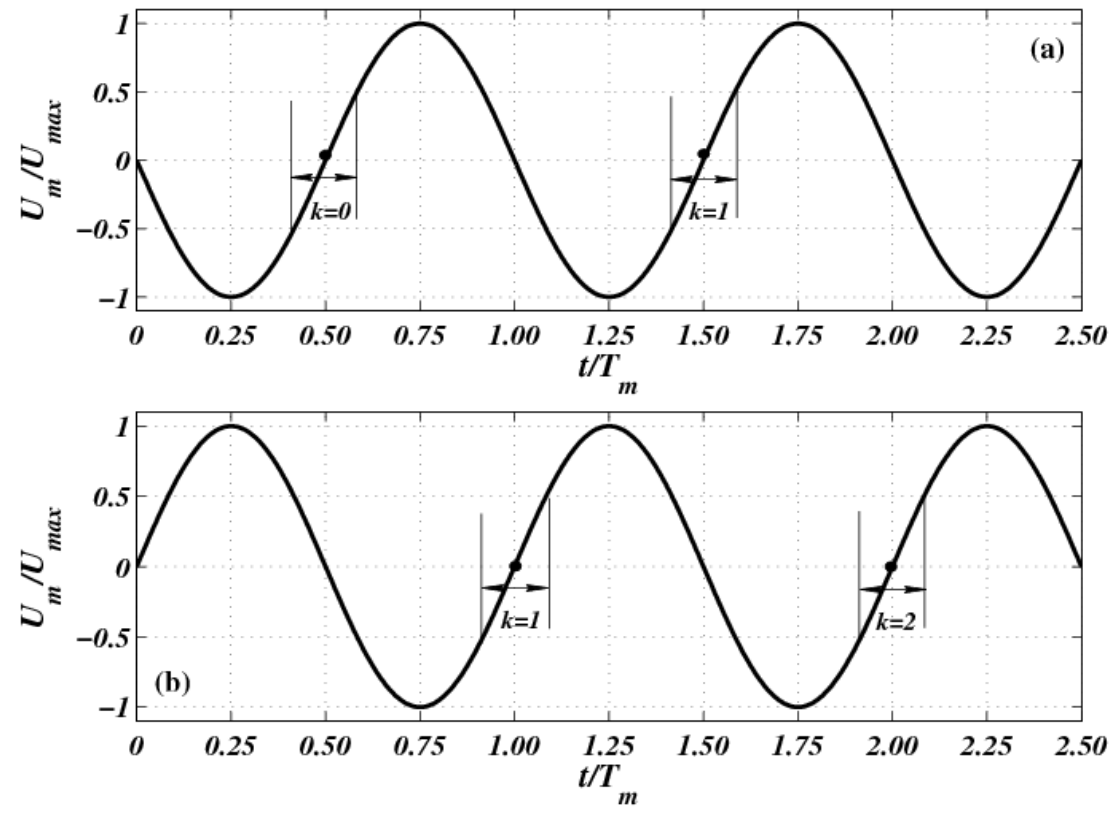

Figure 2. Alternating voltage nodes of (a) $U_{m}(t)=-U_{\max } \sin \left(2 \pi t / T_{m}\right)$, and (b) $U_{m}(t)=U_{\max } \sin \left(2 \pi t / T_{m}\right)$. 


\subsection{Electron-Pulse-Duration Modulation}

The template is used to format your paper and style the text. All margins, column widths, line spaces, and text fonts are prescribed; please do not alter them. You may note peculiarities. For example, the head margin in this template measures proportionately more than is $U_{m}(t)=U_{\max } \sin \left(2 \pi t / T_{m}\right)$. This measurement and others are deliberate, using specifications that anticipate your paper as one part of the entire journals, and not as an independent document. Please do not revise any of the current designations.

To quantitatively define the modulation effect, the transition time for electrons to travel through the pulse modulation region, in the case of no energy modulation, is given

$$
t_{0}\left(\varepsilon_{i}\right)=\sqrt{\frac{m_{e}\left(L+d_{2}\right)^{2}}{2\left(\varepsilon_{i}+e U\right)}} .
$$

With quasi-linear energy modulation, the changes of transition time for electrons in the most leading and the most rear portions can be got as the following, respectively

$$
\begin{aligned}
& \Delta t\left(\varepsilon_{\text {first }}\right)=t_{0}\left(\varepsilon_{\text {first }}\right)-t_{m}\left(\varepsilon_{\text {first }}\right)
\end{aligned}
$$

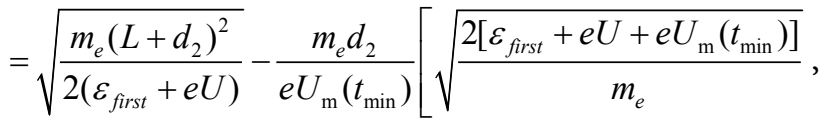

$$
\begin{aligned}
& \left.-\sqrt{\left.\frac{2\left(\varepsilon_{\text {first }}+e U\right)}{m_{e}}\right]}\right]-\sqrt{\frac{m_{e} L^{2}}{2\left[\varepsilon_{\text {first }}+e U+e U_{\mathrm{m}}\left(t_{\min }\right)\right]}} \\
& \Delta t\left(\varepsilon_{\text {last }}\right)=t_{0}\left(\varepsilon_{\text {last }}\right)-t_{\mathrm{m}}\left(\varepsilon_{\text {last }}\right) \\
& =\sqrt{\frac{m_{e}\left(L+d_{2}\right)^{2}}{2\left(\varepsilon_{\text {last }}+e U\right)}}-\frac{m_{e} d_{2}}{e U_{\mathrm{m}}\left(t_{\max }\right)}\left[\sqrt{\frac{2\left[\varepsilon_{\text {last }}+e U+e U_{\mathrm{m}}\left(t_{\max }\right)\right]}{m_{e}}} .\right. \\
& \left.-\sqrt{\left.\frac{2\left(\varepsilon_{\text {last }}+e U\right)}{m_{e}}\right]}\right]-\sqrt{\frac{m_{e} L^{2}}{2\left[\varepsilon_{\text {last }}+e U+e U_{\mathrm{m}}\left(t_{\max }\right)\right]}}
\end{aligned}
$$

So the overall modulation magnitude of electron pulse duration

$$
\Delta t=\Delta t\left(\varepsilon_{\text {last }}\right)-\Delta t\left(\varepsilon_{\text {first }}\right) .
$$

Using the practical parameter setting of $d_{2} \ll L$, expressions (27) and (28) reduce to

$$
\begin{aligned}
& \Delta t\left(\varepsilon_{\text {first }}\right)=\frac{L U_{\mathrm{m}}\left(t_{\min }\right)}{2 U} \sqrt{\frac{1}{2 \eta U}}, \\
& \Delta t\left(\varepsilon_{\text {last }}\right)=\frac{L U_{\mathrm{m}}\left(t_{\max }\right)}{2 U} \sqrt{\frac{1}{2 \eta U}} .
\end{aligned}
$$

With $U_{m}\left(t_{\max }\right)=-U_{m}\left(t_{\min }\right)$ of the prerequisite for symmetric type modulation, expression (28) gives

$$
\Delta t=\frac{L U_{\mathrm{m}}\left(t_{\max }\right)}{U} \sqrt{\frac{1}{2 \eta U}} .
$$




\section{An Example Illustration}

An example of a cylindrically symmetric photoelectron gun system is presented here to verify the method. The geometric parameters as illustrated in Figure 1 and others of the initial electron pulse are as follows: $U=30 \mathrm{kV}, d_{1}=3 \mathrm{~mm}$, $d_{2}=1 \mathrm{~mm}, L=10 \mathrm{~mm}, \tau_{0}=150 \mathrm{fs}, \Delta \varepsilon_{0}=0.2 \mathrm{eV}, r_{0}=0.5 \mathrm{~mm}$. And the expected compressive modulation magnitude on electron pulse duration is $200 \mathrm{fs}$ at least.

With these settings, the parameters of the electron pulse in acceleration region can be got as $t_{\text {min }}=5.8107 \times 10^{11} \mathrm{~s}, t_{\max }=5.8407 \times 10^{11} \mathrm{~s}$ and $t_{\text {mid }}=5.8207 \times 10^{11} \mathrm{~s}$. Using relations determined by expressions (16), (17) and (18) for the case of $U_{m}(t)=-U_{\max } \sin \left(2 \pi t / T_{m}\right), \quad k \leq 31$ can be got and the related parameters, e.g., $T_{m}$, can be achieved for different alternating voltage nodes scope $k$ ranging from 0 to 31 . The case of $k=31$ only is used in the following simulation.

The modulation voltages on both the most leading and the most rear electrons thus is $U_{m}\left(t_{\max }\right)=0.50 U_{\max }$ and $U_{m}\left(t_{\min }\right)=-0.50 U_{\max }$ respectively. With expression (29), solving the inequality of $\Delta t \geq 200 \mathrm{fs}$ gives $U_{\max } \geq 120 \mathrm{~V}$.The compression effect on electron pulse duration is shown in Figure 3. It shows that the initial electron pulse at photocathode is broadened quickly to $\tau=300 \mathrm{fs}$ in photocathode-anode acceleration region, exactly about $0.1 \mathrm{~mm}$ in the vicinity of photocathode, and then undergoes electron-pulse-duration compressive compensation throughout all the modulation region, which is just resulted from the differential energy modulation in electron energy modulation region next to acceleration region. The final electron pulse duration at the target plane is $\tau^{\prime}=100 \mathrm{fs}$ and the overall compressive modulation value is about $200 \mathrm{fs}$.

\section{Conclusion}

We have shown that the proposed technique can provide symmetric type

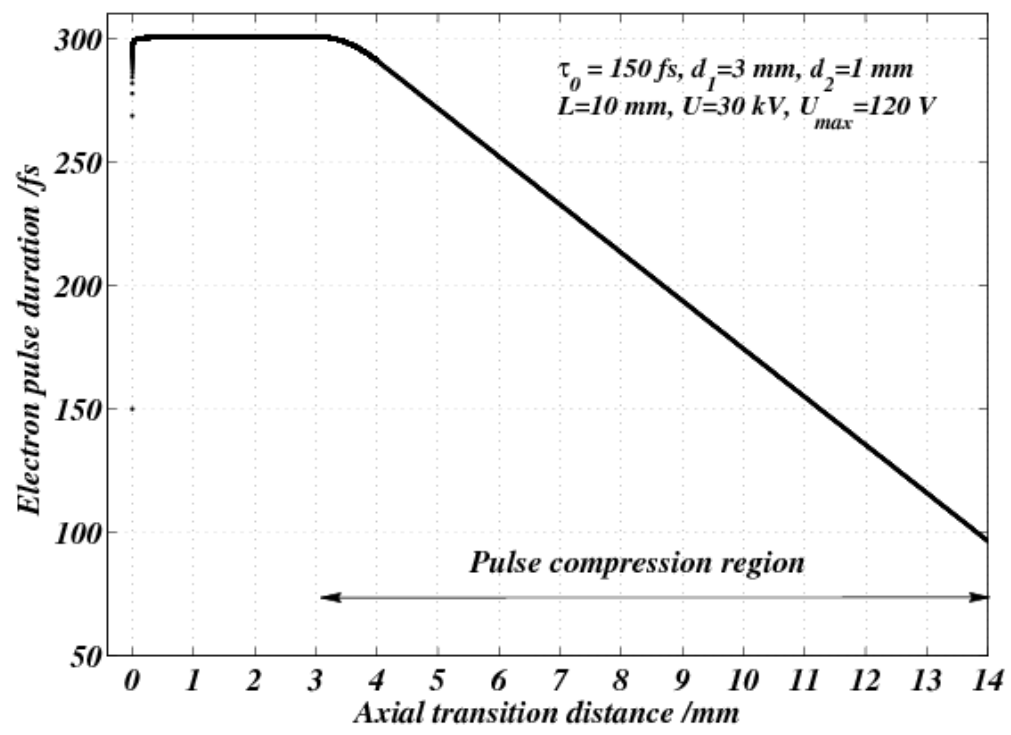

Figure 3. Modulating effect of the alternating electric field on electron pulse duration. 
quasi-linear compensation for electron pulse broadening. This is accomplished by introducing an alternating electric field resonator, which renders the desired quasi-linear differential energy modulation on the electron pulse, with both the leading electrons undergoing negative energy modulation to decelerate and the rear ones positive energy modulation to accelerate. The technical details are demonstrated and the example gives the further illustration.

\section{Acknowledgements}

The work was supported by the National Natural Science Foundation of China (Grant No. 11675258, 11505289).

\section{Conflicts of Interest}

The authors declare no conflicts of interest regarding the publication of this paper.

\section{References}

[1] Kubicki, A.A., Bojarski, P., Grinberg, M., Sasownik, M. and Kuklinski, B. (2006) Time-Resolved Streak Camera System with Solid State Laser and Optical Parametric Generator in Different Spectroscopic Applications. Optics Communications, 263, 275-280. https://doi.org/10.1016/j.optcom.2006.01.043

[2] Reckenthaeler, P., Centurion, M., Fub, W., Trushin, S.A., Krausz, F. and Fill, E.E. (2009) Time-Resolved Electron Diffraction from Selectively Aligned Molecules. Physical Review Letters, 102, 213001/1-213001/4. https://doi.org/10.1103/PhysRevLett.102.213001

[3] Fruhling, U., Wieland, M., Gensch, M., Gebert, T., Schutte, B., Krikunova, M., Kalms, R., Budzyn, F., Grimm, O., Rossbach, J., Plonjes, E. and Drescher, M. (2009) Single-Shot Terahertz-Field-Driven X-Ray Streak Camera. Nature Photonics, 3, 523-528. https://doi.org/10.1038/nphoton.2009.160

[4] Zavoisky, E.K. and Fanchenko, S.D. (1965) Image Converter High-Speed Photography with $10^{-9}-10^{-14}$ Sec Time Resolution. Applied Optics, 4, 1155-1169. https://doi.org/10.1364/AO.4.001155

[5] Niu, H. and Sibbett, W. (1981) Theoretical Analysis of Space Charge Effects in Photochron Streak Cameras. Rev. Sci. Instrum, 52, 1830-1836. https://doi.org/10.1063/1.1136540

[6] Qian, B.L. and Elsayed-Ali, H.E. (2002) Acceleration Element for Femtosecond Electron Pulse Compression. Phys. Rev. E, 65, 046502/1-046502/7. https://doi.org/10.1103/PhysRevE.65.046502

[7] Baum, P. and Zewail, A.H. (2006) Breaking Resolution Limits in Ultrafast Electron Diffraction and Microscopy. ProcNatlAcadSci USA, 103, 16105-16110. https://doi.org/10.1073/pnas.0607451103

[8] Wang, Y.H. and Gedik, N. (2012) Electron Pulse Compression with a Practical Reflectron Design for Ultrafast Electron Diffraction. IEEE Journal of Selected Topics in Quantum Electronics, 18, 140-147. https://doi.org/10.1109/JSTQE.2011.2112339

[9] Fill, E., Veisz, L., Apolonski, A. and Krausz, F. (2006) Sub-fs Electron Pulses for Ultrafast Electron Diffraction. New Journal of Physics, 8, 272/1-272/11.

[10] Veisz, L., Kurkin, G., Chernov, K., Tarnetsky, V., Apolonski, A., Krausz, F. and Fill, 
E. (2007) Hybrid de-ac Electron Gun for fs-Electron Pulse Generation. New Journal of Physics, 9, 451/1-451/17.

[11] Qiang, J., Byrd, J.M., Feng, J. and Huang, G. (2009) X-Ray Streak Camera Temporal Resolution Improvement Using a Longitudinal Time-Dependent Field. Nuclear Instruments and Methods in Physics Research A, 598, 465-469. https://doi.org/10.1016/j.nima.2008.09.030

[12] Hilbert, S.A., Uiterwaal, C., Barwick, B., Batelaan, H. and Zewail, A. (2009) Temporal Lenses for Attosecond and Femtosecond Electron Pulses. ProcNatIAcadSci USA, 106, 10558-10563. https://doi.org/10.1073/pnas.0904912106 